\title{
SUMMARY OF POSTER PRESENTATIONS
}

\section{Belloni, T. et AL. An Unstable Central Disk in GRS $1915+105$}

The mass accretion rate required to create the instability is much larger than accounted for by the radiation emitted at quiescence, and the authors conclude that much energy is advected into the black hole.

Benz, A.O. And G. Paesold Search for Prompt Radio Emission of Gamma-Ray Bursts Non-detections using solar spectrographs would put useful limits on fireball expansion rate if the sources were less than $1 \mathrm{kpc}$ away.

BorozDIN, K.N. ET AL. Observations of X-ray Novae by MIR-KVANT

A number of bright X-ray transients from Nova Vul in 1988 to GRS 1739 - 278 in 1996 displayed characteristics suggestive of black hole accretors.

Casares, J. E.L. Martin, and K. KuUlKers High Resolution Spectroscopy of Cyg $X-3$

The rotation rate of the secondary and the mass ratio of the system have been measured.

Che, H. And Y. Yang A New Test for Cosmological Time Dilation in Long and Short Bursts A parameter called "peakedness" shows evidence for cosmological time dilation of gamma ray bursters.

CherepaschchuK, A.M. Analysis of Optical and Infrared Light Curves of Cyg X-1 Improved values of the orbital elements have been derived from IR and optical light curves.

FALOMo, R. ET AL. The HST Snapshot Survey of BL Lacs

WFPC2 images permit the study of host galaxies and environments of BL Lacs in comparison with other AGN and ground-based studies.

Fraix-Burnet, D. The Gamma Ray/Radio-Vlbi Connection

A two-fluid model for the gamma ray and radio emission of VLBI components of AGNs is presented.

Harris, D.E., W. Junor, And J.D. Silverman Circumstational Evidence for Relativistic Beaming of $X$-rays in a Tailed Radio Galaxy

The circumstance is the absence of both broad and narrow emission line regions in the X-ray spectrum of $26 \mathrm{~W} 20$.

Hubeny, I. And Hubeny, V. Non-Lte Models and Theoretical Spectra of Accretion Disks in Active Galactic Nuclei

Non-LTE effects and a proper treatment of vertical structure are very important for modeling th UV to soft X-ray spectra from AGN disks.

MAKINO, F. ET AL. X-ray Variability of BL Lac Objects

Data are available from a number of missions covering a wide variety of time scales and types of variability.

Martin, E.L., R. Rebolo, and J. Cesares. Lithium in High Energy Transients

Strong LiI features have been seen in at least four X-ray transients and probably results from de-excitation following electron capture on $\mathrm{Be}$. 
MendeZ, M. ET AL. The Kilohertz QPO in $4 U 0614+091$

RXTE data show different frequencies during different observing periods and associated (not necessarily causal) changes in spectrum.

Mendez, M., M. VAn Der Klis, And T. Belloni Source States on GX 339-4

The various states of brightness and spectrum of this black hole candidate probably result from changes in mass accretion rate.

Mitsuda, K. ET AL. ASCA Observations of Soft X-ray Transients in Quiescence

$\mathrm{X} 1608-552$ and Cen $\mathrm{X}-4$ display black body spectra in quiescence. The latter also has a power law component and probably a $32 \mathrm{~Hz}$ periodicity.

PANChENKo, I.E. AND V.M. LIPUNOV The Revival of a Radiopulsar by Gravitational Waves Powerful radio bursts should precede the bursts of gravitational radiation from merging pairs of neutron stars and provide a warning of them.

Robinson, C.R. ET AL. Hard X-ray Observations of X-ray Transients with CGRo/BATSE

Recurrent bursters do not display indentical spectra at each reappearance.

Smit, J.M. ANd M. VAN DER Klis Long Term X-ray Spectral Variability of Cyg $X-3$

EXOSAT data over 190 hours show spectral changes with orbit period and between low and high states, including evidence for branches in the X-ray color-color diagram.

SPENCER, R.E. Radio Emission from X-ray Novae

All $\mathrm{X}$-ray novae are probably radio sources near outburst and modeling suggests massive accretors that are probably black holes.

Tian, W.W. and J.L. Han. Large Pulsars Detected in Nvss

Comparison of two catalogues shows positional and flux consistency over many years for most objects and large proper motions for a couple.

UEDA, Y. ET AL. ASCA Observations of the Superluminal Source GRO J1655-40

Data from 1994 and 1995 show the first clear evidence for absorption lines from an accretionpowered X-ray source, probably to be interpreted as He- or H- like ions of iron.

UnNo, W. ET AL. Wanted!! Galactic Superluminal CR-Accelerating Transients

The small subset of arrival time intervals of cosmic ray air showers that show chaotic behavior may arise in nearby superluminal transients.

van der Hooft, F., M.H.M. Heemskerk, and J. VAN PARAdiJs The Quiescent Optical Light Curve of Nova Scorpii 1994 (GRO J1655-40)

New and archival photometric data set limits to the mass of the accreting black hole.

\section{Wagner, S.J. And C. von Montigny Fast Gamma-Ray Flares from Blazars}

Gamma-rays and radio emission from these are highly variable and the former, at least, may be entirely due to the sum of frequent flares.

VARgAS, M. ET AL. Sigma Observations of X-ray Novae

Analysis of high energy spectra and variability suggests that peak luminosity may be a standard candle.

WANG, Z.-R. The Morphologies of SNRs and their ISM and CSM

Many of these do not much resemble the standard (shell, plerion, etc.) classes as a result of interaction with surrounding material, some of which is due directly to the progenitor star. 
WANG, Z.-R., Q.-Y. QU, AND Y ChEN The AD 393 Guest Star and the SNR RX J1713.7-3946 This is a possible association of another historical SN with an observed remnant.

WiJnands, R.A.D., E. KuUlkers, And A.P. Smale Detection of a Roughly 78 day Period in the Rxte, Vela $5 B$, and Ariel $V$ all Sky Monitor Data of Cygnus $X-2$

The period is quite stable and is probably that of the precession of a tilted accretion disk.

Additional abstracts were submitted by M.E. Prokorov, T.N. Korokhova, P. Groot, M. Guainazzi, M. Kalinkov, G. Pizzichini, S.V. Repin, N.R. Robba, A.V. Serber, and P. Callanan, but could not be scheduled for display owing to late registration, or non-registration, of the authors. 